\title{
Structure and Spectral and Magnetic Properties of a Series of Carbacylamidophosphate Pentanuclear Lanthanide(III) Hydroxo Complexes
}

Nataliia S. Kariaka, ${ }^{1}$ Sergey V. Kolotilov, ${ }^{2}$ Paula Gawryszewska, ${ }^{3}$ Ewa Kasprzycka, ${ }^{3}$ Marek Weselski, ${ }^{3}$ Viktoriya V. Dyakonenko, ${ }^{4}$ Svitlana V. Shishkina, ${ }^{4,5}$ Victor A. Trush,${ }^{1}$ Vladimir M. Amirkhanov*1

1 Taras Shevchenko National University of Kyiv, Department of Chemistry, 64/13, Volodymyrska Street, Kyiv 01601, Ukraine

2 L.V.Pisarzhevskii Institute of Physical Chemistry of the National Academy of Sciences of Ukraine Nauky ave. 31, Kyiv 03028, Ukraine

3 Faculty of Chemistry University of Wroclaw 14 F. Joliot-Curie Str. 50-383 Wroclaw, Poland

$4 \quad$ STC "Institute for Single Crystals", National Academy of Science of Ukraine, Nauky ave. 60, 61001, Kharkiv, Ukraine

$5 \quad$ V. N. Karazin Kharkiv National University 4 Svobody sq., Kharkiv 61122, Ukraine

Table S1. Summary of crystallographic data for[Na2 $\left.\mathbf{L}_{2}\right]_{\mathbf{n}}$ and complexes $\mathbf{1 , 4 , 5}$.

\begin{tabular}{|c|c|c|c|c|}
\hline Compounds & {$\left[\mathbf{N a}_{2} \mathbf{L}_{2}\right]_{\mathbf{n}}$} & $\mathbf{1}$ & 4 & 5 \\
\hline Formula weight & 502.29 & 3087.81 & 3202.91 & 3231.81 \\
\hline Temperature (K) & $294(2)$ & $294(2)$ & 295 & 100 \\
\hline Crystal system & \multicolumn{4}{|c|}{ monoclinic } \\
\hline Space group & $\mathrm{P} 2{ }_{1} / \mathrm{c}$ & & $\mathrm{C} 2 / \mathrm{c}$ & \\
\hline \multicolumn{5}{|l|}{ Unit cell dimensions } \\
\hline $\mathrm{a},(\AA)$ & $7.2740(6)$ & $31.8022(17)$ & $31.8478(19)$ & $31.6098(10)$ \\
\hline $\mathrm{b},(\AA)$ & $11.737(3)$ & $14.6840(6)$ & $14.3424(7)$ & $14.1165(3)$ \\
\hline $\mathrm{c},(\AA)$ & $27.262(2)$ & $28.1549(12)$ & $28.0295(18)$ & 27.4193(9) \\
\hline$\alpha,\left(^{\circ}\right)$ & 90 & 90.00 & 90.00 & 90.00 \\
\hline$\beta,\left(^{\circ}\right)$ & $100.116(7)$ & $112.481(6)$ & $111.873(8)$ & $112.061(4)$ \\
\hline$\gamma,\left(^{\circ}\right)$ & 90 & 90.00 & 90.00 & 90.00 \\
\hline Volume, $\left(\AA^{3}\right)$ & 2291.3(6) & $12148.9(11)$ & $11881.6(12)$ & $11339.3(6)$ \\
\hline $\mathrm{Z}$ & 4 & 4 & 4 & 4 \\
\hline$D_{\text {calc }}\left(\mathrm{g} / \mathrm{cm}^{3}\right)$ & 1.456 & 1.688 & 1.791 & 1.893 \\
\hline$\mu, \mathrm{mm}^{-1}$ & 0.274 & 2.317 & 3.716 & 4.317 \\
\hline $\mathrm{F}(000)$ & 1040 & 6140 & 6300 & 634. \\
\hline $\begin{array}{c}\text { Reflections } \\
\text { collected/independent }\end{array}$ & $21716 / 6634$ & $38822 / 10664$ & $39904 / 10425$ & $43308 / 11138$ \\
\hline Goodness-of-fit on $\mathrm{F}^{2}$ & 1.049 & 0.949 & 0.992 & 1.055 \\
\hline $\begin{array}{l}\text { Final } \mathrm{R} \text { indices } \\
\qquad[I>2 \sigma(I)]\end{array}$ & $\begin{array}{c}\mathrm{R} 1=0.0474, \\
\mathrm{wR} 2=0.1102\end{array}$ & $\begin{array}{c}\mathrm{R} 1=0.0597, \\
\mathrm{wR} 2=0.0969\end{array}$ & $\begin{array}{c}\mathrm{R} 1=0.0488 \\
\mathrm{wR}_{2}=0.1134\end{array}$ & $\begin{array}{c}\mathrm{R} 1=0.0453 \\
\mathrm{wR}_{2}=0.0935\end{array}$ \\
\hline $\mathrm{R}$ indices (all data) & $\begin{array}{c}\mathrm{R} 1=0.0774, \\
\mathrm{wR} 2=0.1218\end{array}$ & $\begin{array}{l}\mathrm{R} 1=0.1472, \\
\mathrm{wR} 2=0.1160\end{array}$ & $\begin{array}{c}\mathrm{R} 1=0.0854 \\
\mathrm{wR}_{2}=0.1285\end{array}$ & $\begin{array}{c}\mathrm{R} 1=0.0680 \\
\mathrm{wR}_{2}=0.1033\end{array}$ \\
\hline CCDC & 1834474 & 1834471 & 1834473 & 1834472 \\
\hline
\end{tabular}


Table S2. Some bond length $(\AA)$ and angles $\left(^{\circ}\right)$ in structure of $\left[\mathbf{N a} \mathbf{2}_{2} \mathbf{L}_{2}\right]_{\mathbf{n}}$

\begin{tabular}{|c|c|c|c|c|c|}
\hline \multicolumn{6}{|c|}{ Bond length $(\AA)$} \\
\hline $\mathrm{P} 1-\mathrm{O} 2$ & $1.486(2)$ & $\mathrm{Na} 1-\mathrm{O} 1(\mathrm{C})$ & $2.552(2)$ & $\mathrm{Na} 2-\mathrm{O} 8(\mathrm{C})$ & $2.579(2)$ \\
\hline $\mathrm{P} 2-\mathrm{O} 5$ & $1.481(2)$ & $\mathrm{Na} 2-\mathrm{O} 1^{\prime}(\mathrm{C})$ & $2.344(2)$ & $\mathrm{Na1-O8^{ \prime }}(\mathrm{C})$ & $2.343(2)$ \\
\hline $\mathrm{C} 1-\mathrm{O} 1$ & $1.248(2)$ & $\mathrm{Na} 1-\mathrm{O} 2(\mathrm{P})$ & $2.364(2)$ & $\mathrm{Na} 2-\mathrm{O} 5(\mathrm{P})$ & $2.361(2)$ \\
\hline C11-O8 & $1.251(2)$ & $\mathrm{Na} 1-\mathrm{O} 2^{\prime}(\mathrm{P})$ & $2.352(2)$ & $\mathrm{Na} 2-5^{\prime}(\mathrm{P})$ & $2.352(2)$ \\
\hline $\mathrm{P} 1-\mathrm{N} 1$ & $1.593(2)$ & $\mathrm{Na} 2-\mathrm{O} 2{ }^{\prime}(\mathrm{P})$ & $2.656(2)$ & $\mathrm{Na} 1-\mathrm{O}^{\prime}(\mathrm{P})$ & $2.712(2)$ \\
\hline $\mathrm{P} 2-\mathrm{N} 2$ & $1.589(3)$ & $\mathrm{Na} 1-\mathrm{O} 6\left(\mathrm{CH}_{3}\right)$ & $2.546(2)$ & $\mathrm{Na} 2-\mathrm{O} 4\left(\mathrm{CH}_{3}\right)$ & $2.560(2)$ \\
\hline C1-N1 & $1.319(2)$ & & & & \\
\hline C11-N2 & $1.319(2)$ & & & & \\
\hline \multicolumn{6}{|c|}{ Angles $\left({ }^{\circ}\right)$} \\
\hline O2-P1-N1 & & $123.34(8)$ & O5-P2-N2 & & $123.70(9)$ \\
\hline $\mathrm{O} 2-\mathrm{P} 1-\mathrm{O} 3$ & & $112.53(8)$ & O5-P2-O6 & & $104.08(8)$ \\
\hline $\mathrm{O} 2-\mathrm{P} 1-\mathrm{O} 4$ & & $104.02(7)$ & $\mathrm{O} 5-\mathrm{P} 2-\mathrm{O} 7$ & & $112.62(8)$ \\
\hline N1-P1-O3 & & 104.07(9) & N2-P2-O6 & & $105.04(8)$ \\
\hline N1-P1-O4 & & $105.24(8)$ & N2-P2-O7 & & $103.65(9)$ \\
\hline O3-P1-O4 & & 106.39(9) & O6-P2-O7 & & $106.47(9)$ \\
\hline O1-C1-N1 & & $126.12(2)$ & O8-C11-N2 & & $125.93(2)$ \\
\hline $\mathrm{O} 1-\mathrm{C} 1-\mathrm{C} 2$ & & $118.88(2)$ & O8-C11-C12 & & $118.67(2)$ \\
\hline $\mathrm{N} 1-\mathrm{C} 1-\mathrm{C} 2$ & & $114.98(19)$ & $\mathrm{N} 2-\mathrm{C} 11-\mathrm{C} 12$ & & $115.32(2)$ \\
\hline
\end{tabular}

Table S3. Angles $\left(^{\circ}\right)$ of geometrical polyhedra of $\mathrm{Na} 1$ and $\mathrm{Na} 2$ atoms in $\left[\mathbf{N a} 2 \mathbf{L}_{2}\right]_{\mathbf{n}}\left({ }^{1} 2-\mathrm{x},-1-\mathrm{y},-\right.$ $\left.\mathrm{z} ;{ }^{2} 1+\mathrm{x},+\mathrm{y},+\mathrm{z} ;{ }^{3} 1-\mathrm{x},-1-\mathrm{y},-\mathrm{z} ;{ }^{4}-1+\mathrm{x},+\mathrm{y},+\mathrm{z}\right)$

\begin{tabular}{|c|c|c|c|}
\hline O1-Na1-O5 & $70.55(5)$ & $\mathrm{O} 1-\mathrm{Na} 2-\mathrm{O} 4^{4}$ & $96.49(6)$ \\
\hline $\mathrm{O} 2^{1}-\mathrm{Na} 1-\mathrm{O} 1$ & $134.64(6)$ & $\mathrm{O} 1-\mathrm{Na} 2-\mathrm{O}^{3}$ & $101.85(6)$ \\
\hline $\mathrm{O} 2-\mathrm{Na} 1-\mathrm{O} 1$ & $80.91(5)$ & $\mathrm{O} 1-\mathrm{Na} 2-\mathrm{O} 5$ & $80.58(6)$ \\
\hline $\mathrm{O} 2{ }^{1}-\mathrm{Na} 1-\mathrm{O} 2$ & $86.02(5)$ & $\mathrm{O} 1-\mathrm{Na} 2-\mathrm{O} 8$ & $115.81(6)$ \\
\hline O2-Na1-O5 & $128.15(5)$ & $\mathrm{O}_{4}^{4}-\mathrm{Na} 2-\mathrm{O} 2^{4}$ & $55.35(4)$ \\
\hline $\mathrm{O} 2^{1}-\mathrm{Na} 1-\mathrm{O} 5$ & $84.70(5)$ & $\mathrm{O} 4^{4}-\mathrm{Na} 2-\mathrm{O} 8$ & $95.86(5)$ \\
\hline $\mathrm{O} 2^{1}-\mathrm{Na} 1-\mathrm{O} 6$ & $99.81(6)$ & $\mathrm{O}^{3}-\mathrm{Na} 2-\mathrm{O} 2^{4}$ & $85.96(5)$ \\
\hline O6-Na1-O1 & $95.98(6)$ & $\mathrm{O} 5-\mathrm{Na} 2-\mathrm{O} 2^{4}$ & $127.23(5)$ \\
\hline O6-Na1-O5 & $54.69(4)$ & $\mathrm{O}^{3}-\mathrm{Na} 2-\mathrm{O} 4^{4}$ & $99.74(5)$ \\
\hline $\mathrm{O}^{2}-\mathrm{Na} 1-\mathrm{O} 1$ & $116.22(6)$ & $\mathrm{O} 5^{3}-\mathrm{Na} 2-\mathrm{O} 5$ & $86.75(5)$ \\
\hline $\mathrm{O} 8^{2}-\mathrm{Na} 1-\mathrm{O} 2^{1}$ & $103.82(6)$ & $\mathrm{O} 5-\mathrm{Na} 2-\mathrm{O} 8$ & $80.19(5)$ \\
\hline $\mathrm{O}^{2}-\mathrm{Na} 1-\mathrm{O} 2$ & $80.17(6)$ & $\mathrm{O}^{3}-\mathrm{Na} 2-\mathrm{O} 8$ & $137.07(6)$ \\
\hline $\mathrm{O} 8^{2}-\mathrm{Na} 1-\mathrm{O} 6$ & $96.69(6)$ & $\mathrm{O} 8-\mathrm{Na} 2-\mathrm{O} 2^{4}$ & $70.75(4)$ \\
\hline
\end{tabular}

Table S4.The conformational characteristics of six-membered cycles in structures $\left[\mathrm{Na}_{2} \mathbf{L}_{2}\right]_{\mathbf{n}}, \mathbf{1 ,} \mathbf{4 , 5}$. 


\begin{tabular}{|c|c|c|c|c|c|c|}
\hline \multirow[b]{2}{*}{ Structure } & \multirow[b]{2}{*}{ six-membered cycle } & \multicolumn{3}{|c|}{ Puckering parameters* } & \multirow[t]{2}{*}{ Conformation } & \multirow[t]{2}{*}{$\begin{array}{c}\text { Deviations from the } \\
\text { mean plane } \\
\text { (atom/dev., } \AA \text { ) }\end{array}$} \\
\hline & & $\mathrm{S}$ & $\psi, \circ$ & $\theta,^{\circ}$ & & \\
\hline \multirow[t]{2}{*}[\mathbf{Na}_{2}\mathbf{L}_{2}]{$_{\mathbf{n}}$} & Na1-O2-P1-N1-C1-O1 & 0.81 & 34.14 & 67.13 & Twist-boat & $\begin{array}{r}\mathrm{O} 2-0.76 \\
\mathrm{Na} 1-1.99 \\
\end{array}$ \\
\hline & $\mathrm{Na} 2-\mathrm{O} 5-\mathrm{P} 2-\mathrm{N} 2-\mathrm{C} 11-\mathrm{O} 8$ & 0.8 & 34.5 & 64.14 & Twist-boat & $\begin{array}{c}\text { O8 }-1.37 \\
\text { C11 }-0.67 \\
\end{array}$ \\
\hline \multirow[t]{4}{*}{1} & Nd1-O8-C10-N2-P2-O9 & 0.11 & 6.84 & 51.13 & Sofa & $\mathrm{Nd} 10.2$ \\
\hline & Nd2-O13-P3-N1-C19-O12 & 0.52 & 47.01 & 65.53 & Twist-boat & $\begin{array}{ll}\mathrm{O} 13 & 0.48 \\
\mathrm{Nd} 2 & 1.12 \\
\end{array}$ \\
\hline & $\mathrm{Nd} 2-\mathrm{O} 20-\mathrm{C} 37-\mathrm{N} 5-\mathrm{P} 5-\mathrm{O} 21$ & 0.15 & 47.2 & 56.38 & Sofa & $\mathrm{Nd} 2-0.21$ \\
\hline & Nd3-O16-C28-N4-P4-O17 & 0.42 & 49.2 & 56.14 & Sofa & $\mathrm{Nd} 3-0.49$ \\
\hline \multirow[t]{5}{*}{4} & Er1-O1-C1-N1-P1-O4 & 0.43 & 44.74 & 35.40 & Half-chair & $\begin{array}{c}\text { O1 } 0.25 \\
\text { Er1 }-0.31 \\
\end{array}$ \\
\hline & Er2-O6-P2-N2-C10-O5 & 0.46 & 39.64 & 51.98 & Twist-boat & Er2 -0.65 \\
\hline & Er2-O9-C19-N3-P3-O10 & 0.11 & 53.24 & 49.05 & Sofa & Er2 0.23 \\
\hline & Er3-O16-P4-N4-C28-O17 & 0.18 & 20.75 & 45.85 & Sofa & Er3 -0.26 \\
\hline & Er3-O21-C37-N5-P5-O20 & 0.34 & 45.44 & 51.61 & Sofa & N5 -0.22 \\
\hline \multirow[t]{5}{*}{5} & Yb1-O1-C1-N1-P1-O4 & 0.31 & 56.41 & 63.81 & Sofa & Yb1 -0.38 \\
\hline & Yb1-O5-C10-N2-P2-O6 & 0.17 & 3.72 & 38.03 & Sofa & Yb1 0.25 \\
\hline & Yb2-O12-C19-N3-P3-O9 & 0.10 & 8.25 & 47.90 & Sofa & Yb2 0.16 \\
\hline & Yb2-O16-C28-N4-P4-O13 & 0.50 & 53.91 & 34.49 & Half-chair & 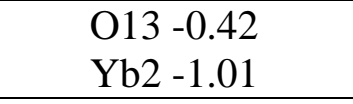 \\
\hline & Yb3-O19-P5-N5-C37-O22 & 0.5 & 33.76 & 82.49 & Twist-boat & $\begin{array}{c}\text { C37 } 0.42 \\
\text { N5 } 0.55 \\
\end{array}$ \\
\hline
\end{tabular}

* According to N. S. Zefirov, V. A. Palyulin, E. E. Dashevskaya, J. Phys. Org. Chem., 1990, 3, 147-158.

Table S5. Criteria of coordination polyhedron determination

\begin{tabular}{|c|c|c|c|c|c|c|}
\hline \multirow[t]{2}{*}{ Polyhedron } & \multicolumn{6}{|c|}{ Angle $\left(^{\circ}\right)$} \\
\hline & $\delta_{1}$ & $\delta_{2}$ & $\delta_{3}$ & $\delta_{4}$ & $\varphi$ & $\omega$ \\
\hline \multicolumn{7}{|c|}{ Theoretical polyhedra for coordination number $8 *$} \\
\hline Dodecahedron, $\mathrm{D}_{2 \mathrm{~d}}$ & 29.5 & 29.5 & 29.5 & 29.5 & 0 & 90 \\
\hline Bicappedtrigonal prism, $\mathrm{C}_{2 \mathrm{v}}$ & 0 & 21.7 & 48.2 & 48.2 & 16.1 & - \\
\hline Square antiprism, $\mathrm{D}_{4 \mathrm{~d}}$ & 0 & 0 & 52.5 & 52.5 & 24.7 & 79.3 \\
\hline \multicolumn{7}{|c|}{ Polyhedra of $\mathrm{Ln}^{\mathrm{III}}$ ions in the complexes } \\
\hline Nd1 & 5.3 & 22.0 & 44.9 & 44.9 & $12.9 ; 33.4$ & 80.9 \\
\hline $\mathrm{Nd} 2$ & 11.1 & 20.6 & 42.5 & 46.4 & $11.2 ; 30.2$ & 81.3 \\
\hline $\mathrm{Nd} 3$ & 0.8 & 8.5 & 51.5 & 51.5 & $21.8 ; 22.8$ & 84.1 \\
\hline Er1 & 0.8 & 3.8 & 47.6 & 47.6 & $21.8 ; 24.3$ & 81.1 \\
\hline Er2 & 9.6 & 18.3 & 43.6 & 46.0 & $16.9 ; 24.8$ & 78.7 \\
\hline Er3 & 6.7 & 21.8 & 43.0 & 43.3 & $13.7 ; 17.6$ & 80.2 \\
\hline Yb1 & 6.9 & 22.7 & 42.3 & 42.7 & 13,$5 ; 17.6$ & 80.3 \\
\hline Yb2 & 12.3 & 17.8 & 43.2 & 46.0 & $15.5 ; 23.9$ & 78.8 \\
\hline Yb3 & 0.9 & 9.0 & 47.6 & 47.6 & $20.7 ; 23.2$ & 82.0 \\
\hline
\end{tabular}

*According to M.A. Porai-Koshits and L.A. Aslanov, J. Struct. Chem.,1972, 13, 244-253. 
Table S6. Selected geometrical parameters in the complexes

\begin{tabular}{|c|c|c|c|}
\hline & 1 & 4 & 5 \\
\hline \multicolumn{4}{|c|}{ Bond lengths ranges $(\AA)$} \\
\hline $\mathrm{Ln}-\mathrm{O}(\mathrm{C})$ & $2.330(6)-2.405(5)$ & $2.246(5)-2.333(4)$ & $2.229(5)-2.313(5)$ \\
\hline $\mathrm{Ln}-\mathrm{O}(\mathrm{P})$ & $2.376(5)-2.583(5)$ & $2.294(5)-2.399(4)$ & $2.271(4)-2.362(4)$ \\
\hline $\mathrm{Ln}-\mathrm{OH}$ & $2.384(5)-2.6975(13)$ & $2.265(4)-2.6180(11)$ & $2.247(4)-2.5918(13)$ \\
\hline $\mathrm{P}-\mathrm{O}$ & $1.470(5)-1.497(4)$ & $1.467(6)-1.533(4)$ & $1.490(6)-1.520(6)$ \\
\hline $\mathrm{P}-\mathrm{N}$ & $1.578(6)-1.588(6)$ & $1.582(6)-1.595(6)$ & $1.601(6)-1.611(6)$ \\
\hline $\mathrm{O}-\mathrm{C}$ & $1.255(7)-1.273(7)$ & $1.233(7)-1.276(10)$ & $1.247(6)-1.272(7)$ \\
\hline $\mathrm{N}-\mathrm{C}$ & $1.304(7)-1.310(7)$ & $1.288(11)-1.327(10)$ & $1.299(13)-1.339(9)$ \\
\hline \multicolumn{4}{|c|}{ Ln - Ln distances $(\AA)$} \\
\hline $\begin{array}{l}\mathrm{Ln}-\mathrm{Ln} \text { (in the } \mathrm{Ln}_{4} \\
\text { basis of pyramide) }\end{array}$ & $3.762-3.768$ & $3.582-3.597$ & $3.535-3.556$ \\
\hline $\begin{array}{l}\text { Ln - Ln (from basal } \\
\text { LnIIIto apical) }\end{array}$ & $3.953-3.954$ & $3.775-3.778$ & $3.735-3.739$ \\
\hline \multicolumn{4}{|c|}{ Angles ranges $\left(^{\circ}\right)$} \\
\hline $\mathrm{C}-\mathrm{O}-\mathrm{Ln}$ & $131.7(6)-140.9(6)$ & $133.8(4)-141.8(4)$ & $132.8(4)-141.9(4)$ \\
\hline $\mathrm{P}-\mathrm{O}-\mathrm{Ln}$ & $127.5(3)-132.6(4)$ & $127.0(2)-132.6(3)$ & 127.34(19)-134.2(3) \\
\hline $\mathrm{O}-\mathrm{Ln}-\mathrm{O}$ & $71.65(19)-75.56(17)$ & $73.78(17)-79.11(15)$ & $74.39(14)-80.00(13)$ \\
\hline $\mathrm{O}-\mathrm{C}-\mathrm{N}$ & $127.2(8)-128.7(9)$ & $126.0(6)-128.0(6)$ & $124.6(6)-128.1(5)$ \\
\hline $\mathrm{O}-\mathrm{P}-\mathrm{N}$ & $118.3(4)-120.8(4)$ & $117.4(4)-119.5(3)$ & $115.9(4)-122.3(4)$ \\
\hline $\mathrm{C}-\mathrm{N}-\mathrm{P}$ & $122.5(6)-128.1(6)$ & $121.1(5)-128.8(4)$ & $121.2(5)-125.5(4)$ \\
\hline
\end{tabular}


Table S7.Relative integral intensities of the $4 f-4 f$ transitions in the absorption spectra of $\mathbf{1 , 2}, \mathbf{4}$ and 5 at $4 \mathrm{~K}$ is lower than at $300 \mathrm{~K}$.

\begin{tabular}{|c|c|c|}
\hline $4 f-4 f$ transitions & Relative integ & al intensities \\
\hline Complex 1 & $\mathrm{~T}=4 \mathrm{~K}$ & $\mathrm{~T}=298 \mathrm{~K}$ \\
\hline${ }^{4} \mathrm{I}_{9 / 2}-{ }^{2} \mathrm{P}_{1 / 2}$ & 0.12 & 0.19 \\
\hline${ }^{4} \mathrm{I}_{9 / 2}-{ }^{2} \mathrm{~K}_{15 / 2},{ }^{4} \mathrm{G}_{11 / 2},{ }^{2} \mathrm{D}_{3 / 2},{ }^{2} \mathrm{G}_{9 / 2}$ & 0.39 & 1.30 \\
\hline${ }^{4} \mathrm{I}_{9 / 2}-{ }^{4} \mathrm{G}_{7 / 2}$ & 1.79 & 5.15 \\
\hline${ }^{4} \mathrm{I}_{9 / 2}-{ }^{2} \mathrm{G}_{7 / 2},{ }^{2} \mathrm{G}_{5 / 2}$ & 5.24 & 15.68 \\
\hline${ }^{4} \mathrm{I}_{9 / 2}-{ }^{4} \mathrm{~S}_{3 / 2},{ }^{4} \mathrm{~F}_{5 / 2}$ & 3.46 & 9.44 \\
\hline${ }^{4} \mathrm{I}_{9 / 2}-{ }^{2} \mathrm{H}_{9 / 2},{ }^{4} \mathrm{~F}_{5 / 2}$ & 4.79 & 11.00 \\
\hline Complex 2 & $\mathrm{~T}=4 \mathrm{~K}$ & $\mathrm{~T}=298 \mathrm{~K}$ \\
\hline${ }^{6} \mathrm{H}_{15 / 2}-{ }^{6} \mathrm{P}_{3 / 2},{ }^{4} \mathrm{M}_{17 / 2}$ & 2.27 & 2.24 \\
\hline${ }^{6} \mathrm{H}_{15 / 2}-{ }^{6} \mathrm{P}_{7 / 2},\left({ }^{4} \mathrm{M},{ }^{4} \mathrm{I}\right)_{15 / 2},\left({ }^{4} \mathrm{~F},{ }^{4} \mathrm{D}\right)_{5 / 2}$ & 3.54 & 5.56 \\
\hline${ }^{6} \mathrm{H}_{15 / 2}-\left({ }^{4} \mathrm{P},{ }^{4} \mathrm{D}\right)_{3 / 2},{ }^{6} \mathrm{P}_{5 / 2},{ }^{4} \mathrm{I}_{11 / 2}$ & 0.70 & 0.70 \\
\hline${ }^{6} \mathrm{H}_{15 / 2}-{ }^{4} \mathrm{~F}_{7 / 2},{ }^{4} \mathrm{I}_{132},{ }^{4} \mathrm{~K}_{17 / 2},\left({ }^{4} \mathrm{M}\right)_{21 / 2,19 / 2}$ & 1.03 & 1.08 \\
\hline${ }^{6} \mathrm{H}_{15 / 2}-{ }^{4} \mathrm{G}_{11 / 2}$ & 1.88 & 2.32 \\
\hline${ }^{6} \mathrm{H}_{15 / 2}-{ }^{4} \mathrm{I}_{15 / 2}$ & 3.79 & 5.86 \\
\hline${ }^{6} \mathrm{H}_{15 / 2}-{ }^{4} \mathrm{~F}_{9 / 2}$ & 0.77 & 1.03 \\
\hline${ }^{6} \mathrm{H}_{15 / 2}-{ }^{6} \mathrm{~F}_{3 / 2}$ & 3.25 & 3.95 \\
\hline${ }^{6} \mathrm{H}_{15 / 2}-{ }^{6} \mathrm{~F}_{5 / 2}$ & 1.16 & 1.22 \\
\hline Complex 4 & $\mathrm{~T}=4 \mathrm{~K}$ & $\mathrm{~T}=298 \mathrm{~K}$ \\
\hline${ }^{4} \mathbf{I}_{15 / 2}-{ }^{2} \mathrm{~K}_{9 / 2}$ & 0.97 & 1.95 \\
\hline${ }^{4} \mathrm{I}_{15 / 2}-{ }^{4} \mathrm{G}_{11 / 2}$ & 3.16 & 5.52 \\
\hline${ }^{4} \mathrm{I}_{15 / 2}-{ }^{2} \mathrm{H}_{9 / 2}$ & 1.91 & 3.26 \\
\hline${ }^{4} \mathrm{I}_{15 / 2}-{ }^{4} \mathrm{~F}_{3 / 2},{ }^{4} \mathrm{~F}_{5 / 2}$ & 5.48 & 5.43 \\
\hline${ }^{4} \mathrm{I}_{15 / 2}-{ }^{4} \mathrm{~F}_{7 / 2}$ & 0.35 & 0.46 \\
\hline${ }^{4} \mathbf{I}_{15 / 2}-{ }^{2} \mathrm{H}_{11 / 2}$ & 1.22 & 1.37 \\
\hline${ }^{4} \mathbf{I}_{15 / 2}-{ }^{4} S_{3 / 2}$ & 0.51 & 0.50 \\
\hline${ }^{4} \mathrm{I}_{15 / 2}-{ }^{4} \mathrm{~F}_{9 / 2}$ & 0.75 & 1.48 \\
\hline${ }^{4} \mathrm{I}_{15 / 2}-{ }^{4} \mathrm{I}_{9 / 2}$ & 4.46 & 6.49 \\
\hline Complex 5 & $\mathrm{~T}=4 \mathrm{~K}$ & $\mathrm{~T}=298 \mathrm{~K}$ \\
\hline${ }^{2} \mathrm{~F}_{7 / 2}-{ }^{2} \mathrm{~F}_{5 / 2}$ & 6.38 & 8.07 \\
\hline
\end{tabular}
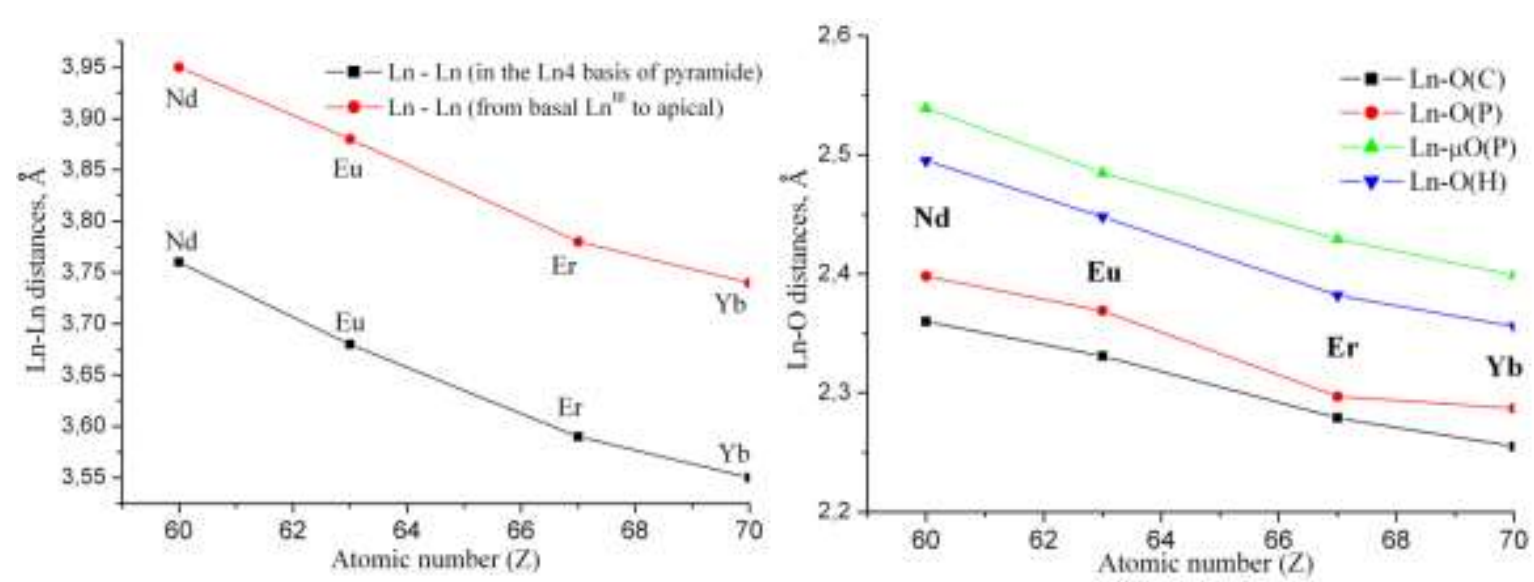

Figure S1. The dependencies of Ln-O bonds lengths and Ln-Ln distances on the atomic number of the lanthanide 

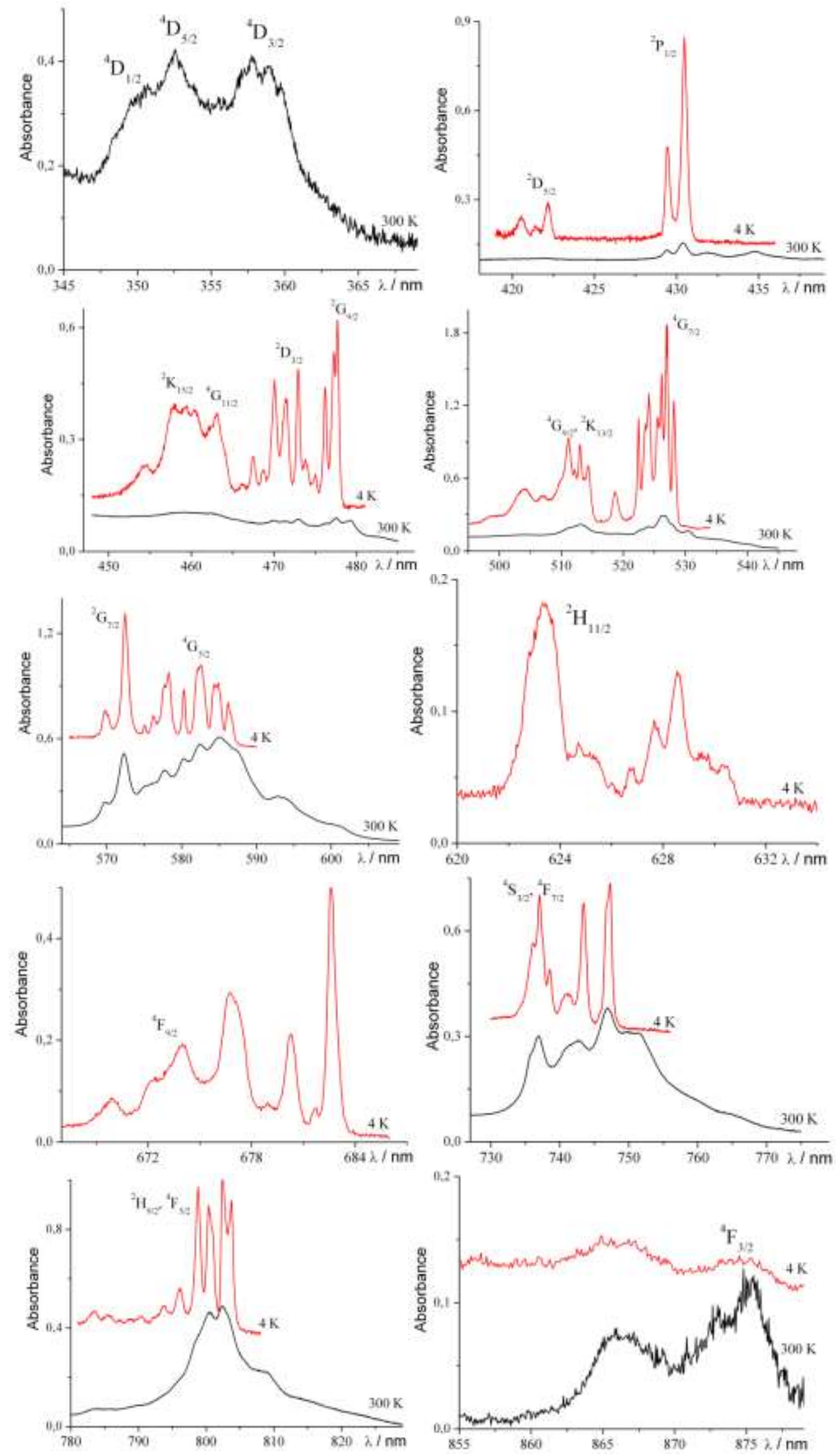

Figure S2. 300 and $4 \mathrm{~K}$ absorption spectra of complex 1. 

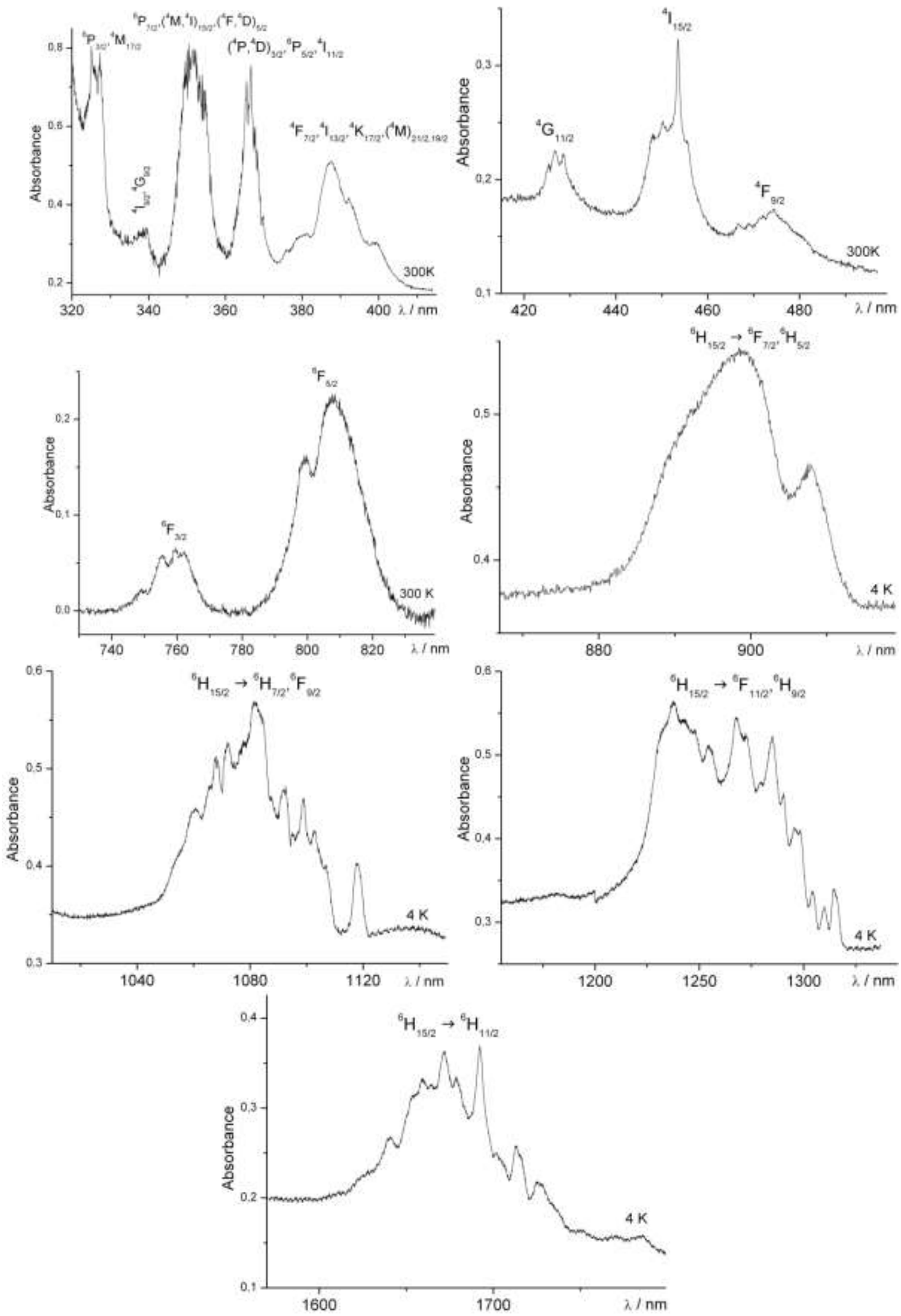

Figure S3. 300 and $4 \mathrm{~K}$ absorption spectra of complex 2. 

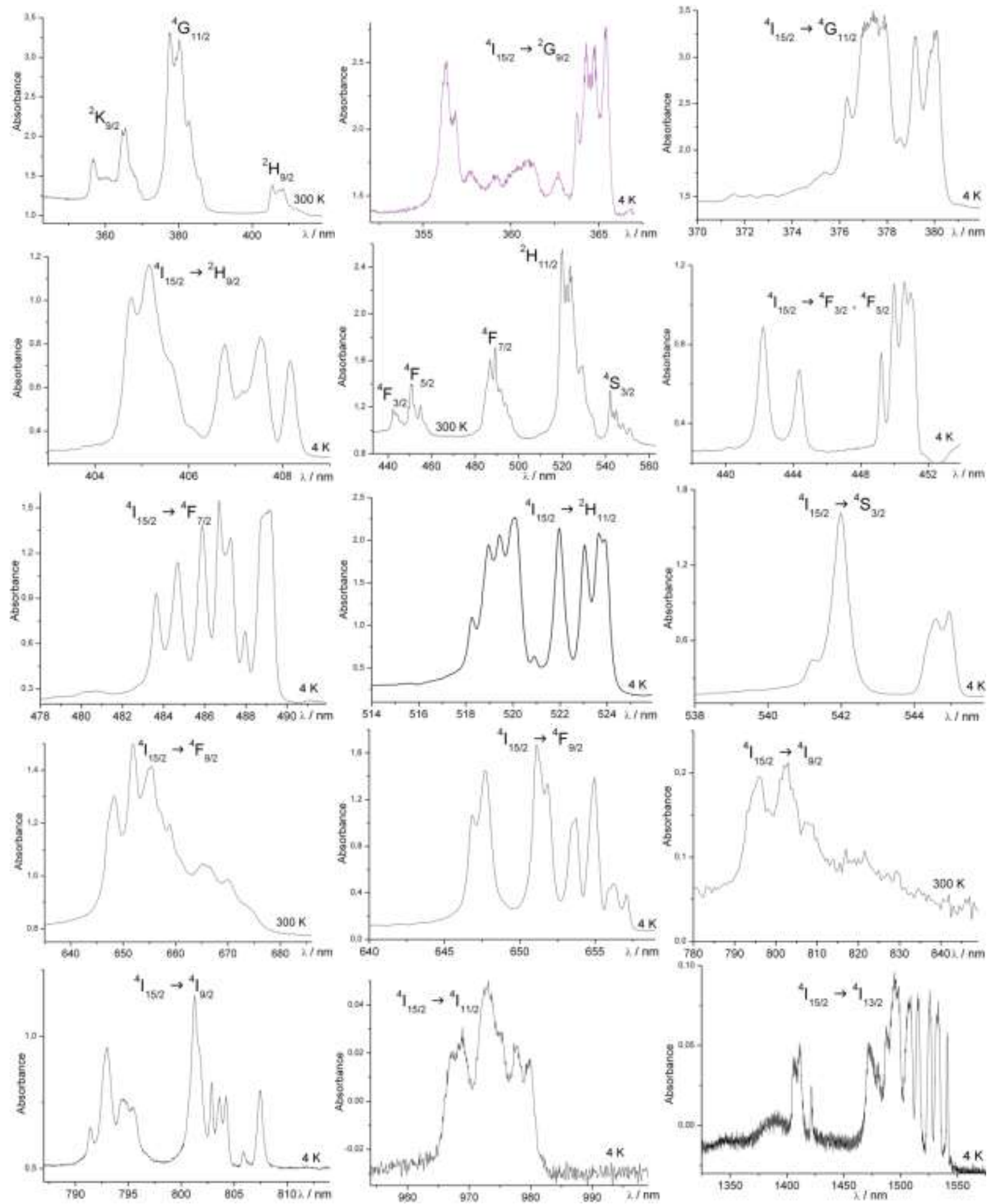

Figure S4. 300 and 4 K absorption spectra of complex 4. 


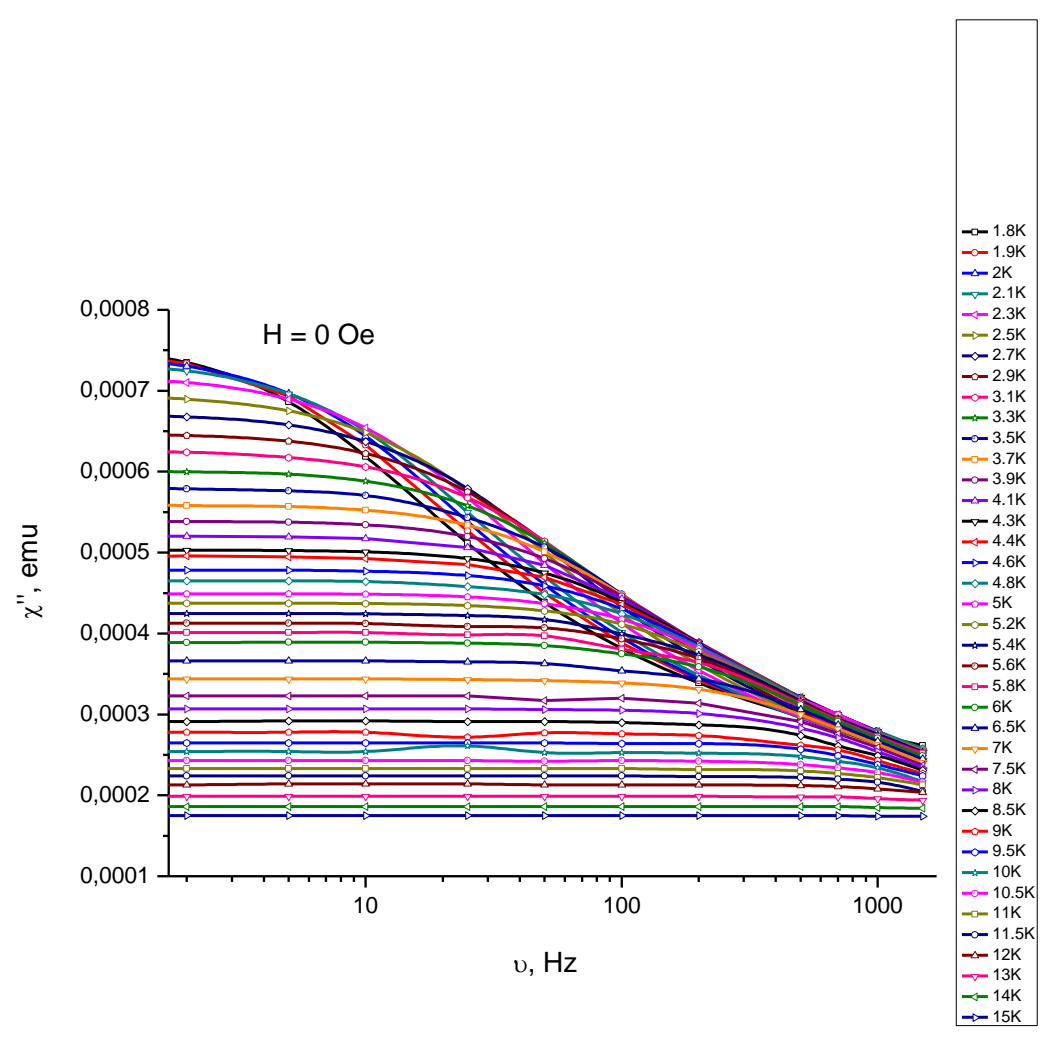

(a)

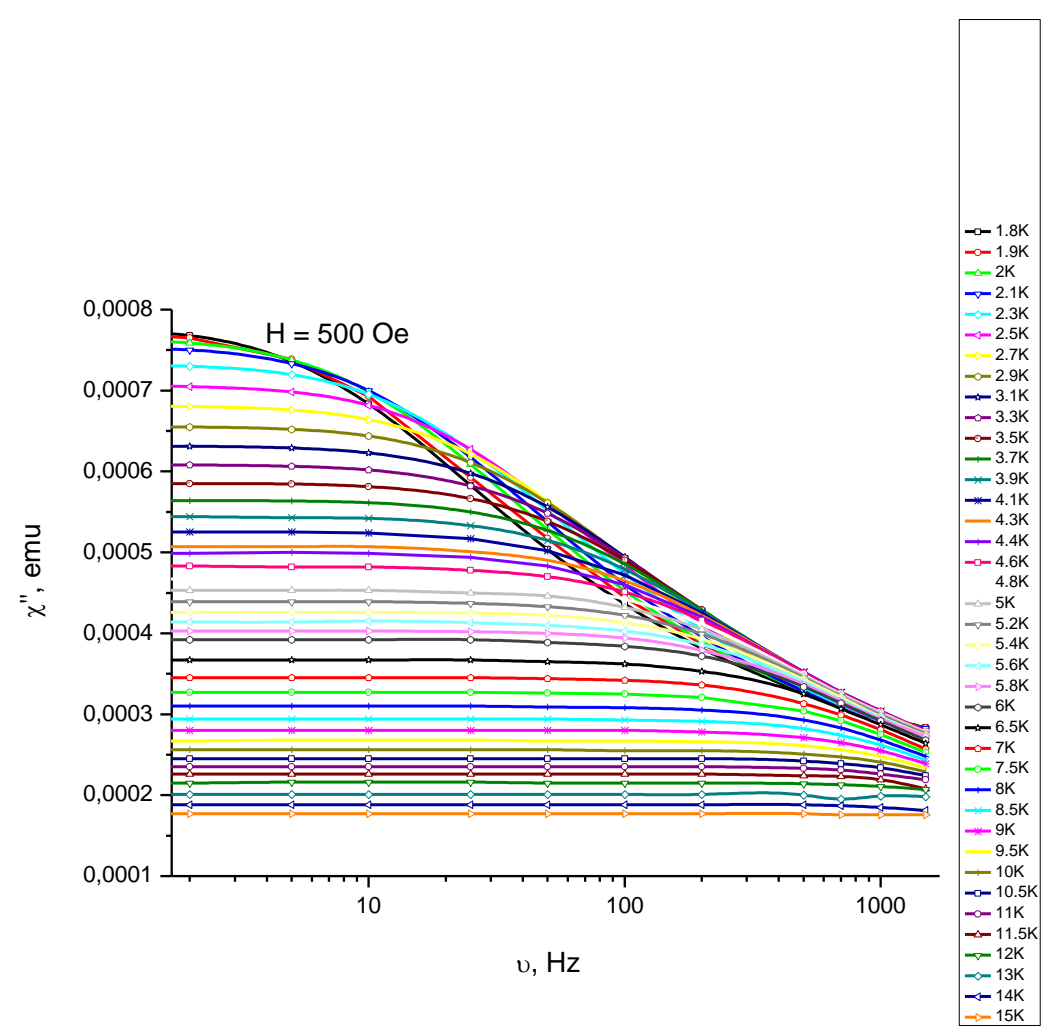

(b)

Figure S5. Frequency dependence of $\chi^{\prime}$ at different temperatures in zero (a) and 500 Oe (b) external DC magnetic fields for 2 . 


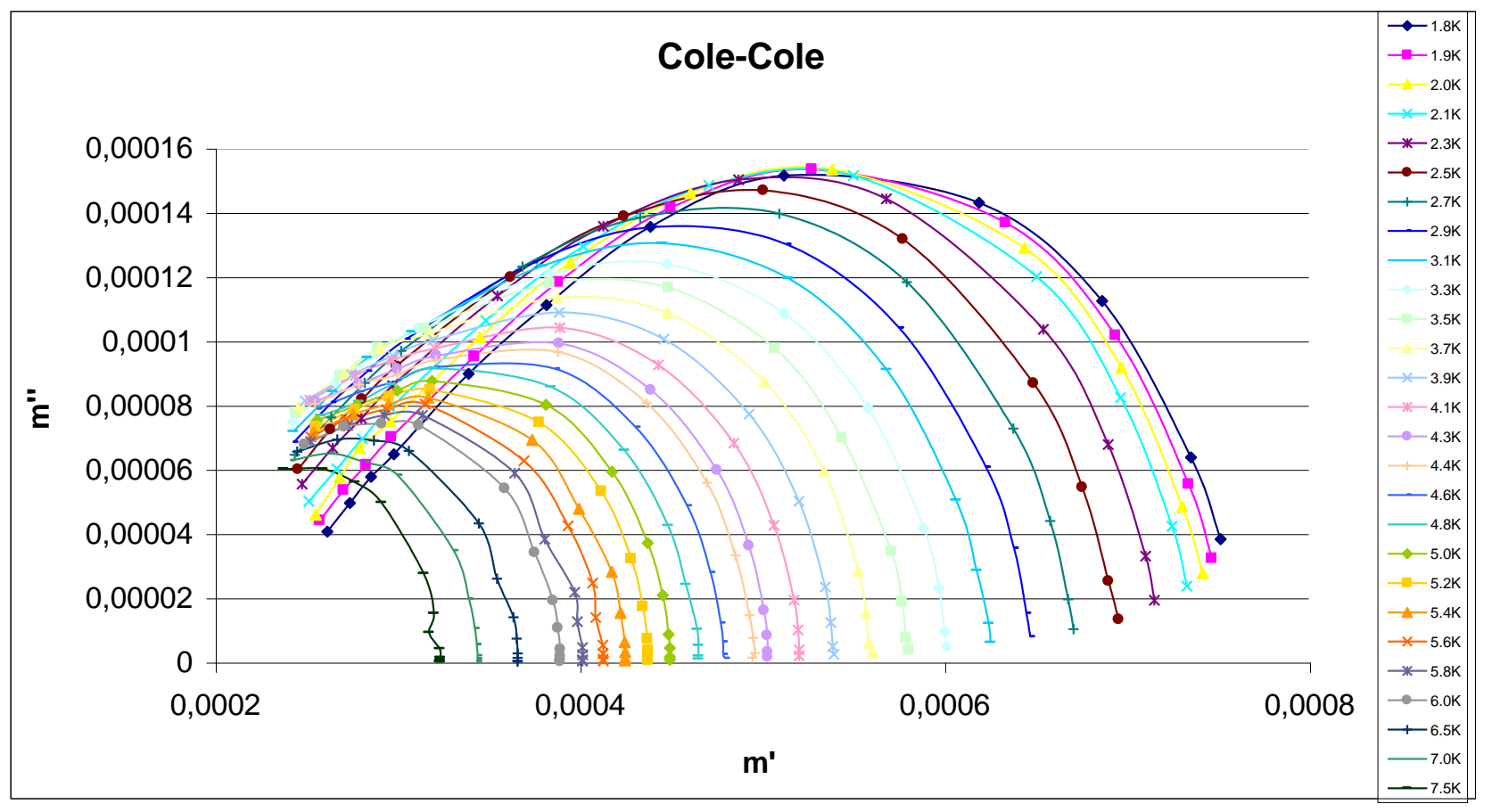

Figure S6. Cole-Cole plot for 2. 